\title{
Intermediate ultrasound guided regional anaesthesia training - recognising a gap in the market
}

Dr John O'Donoghue (ST7), Dr G Lowe (Consultant), Dr D Raj (Consultant)

Queen Elizabeth University Hospital, Anaesthetic Dept, NHS Greater Glasgow \& Clyde, UK

\section{Introduction}

Since their first publication in the mid-1990's, simple ultrasound guided regional anaesthesia techniques have gained increasing popularity and have become integral to the Royal College of Anaesthetists [RCOA] training curriculum. ${ }^{1}$ The techniques offer increased patient satisfaction, reduce the incidence of some complications (such as local anaesthetic systemic toxicity, hemidiaphragmatic paresis, and pneumothorax), and, unlike anatomical techniques, take account of the natural variability of human anatomy. ${ }^{1,2}$

Education and training are essential to develop these skills. There is widespread access to basic workshops at various meetings and advanced cadaveric workshops are also available at a cost of circa $£ 600$. Supervised theatre time with experienced regional anaesthetists is a valuable and cost effective way to develop skills but, unfortunately, trainees' exposure to this is dependent on hospital placements, list allocation, and on-call/service provision. As limited study budgets are available to trainees, those who do not want to subspecialise in regional anaesthesia may be reluctant to spend a large proportion of their budget on an advanced workshop.

\section{Method}

We developed a course that was beyond the scope of basic workshops and would appeal to intermediate level trainees (CT2 to ST5) as either a stepping stone to an advanced workshop or as a cost-effective alternative for trainees who sought to develop their skills further.

Our course ran over one day, a course program was created and mapped to the RCOA curriculum and matrix. Topics we covered with scanning of live models included:- Supraclavicular block - Midhumeral blocks - Foream blocks • Quadratum Lumborum - TAP block • Ilioinguinal block - Sciatic/Popliteal block • Ankle block. In order to distinguish the course from a basic workshop we delivered lecture-based teaching on only one topic (the revision of 'Physics and ultrasound "Knobology"') whilst other topics such as anticoagulation, consent, and complications were dealt with by the discussion of interactive cases. We also had a phantom station that was available for candidates to utilise all day to practice needling skills.

We created pre-course materials for all the live model stations and the lecture/discussion sessions, these were issued to candidates in advance for private study. This allowed more focus on 'hands-on' time, scanning of live models on the day. All scanning supervision was delivered by experienced consultant regional anaesthetists. We also limited the number of candidates to 15 people as this meant that no more than 3 candidates would be at a station, thus all candidates had adequate time to practice scanning and communicate any questions or advice from faculty. At the end of the course, testing took the form of a scanning assessment station to find whether skills and knowledge were gained and retained.

We applied and were approved for 5 external RCOA continuing professional development points. The cost of the course was $£ 125$ and the course was oversubscribed. We used a google form in order to acquire anonymous feedback from our candidates.
Results

The feedback suggested that the course was a great success. Course content: The pre-course material was helpful and appropriate.

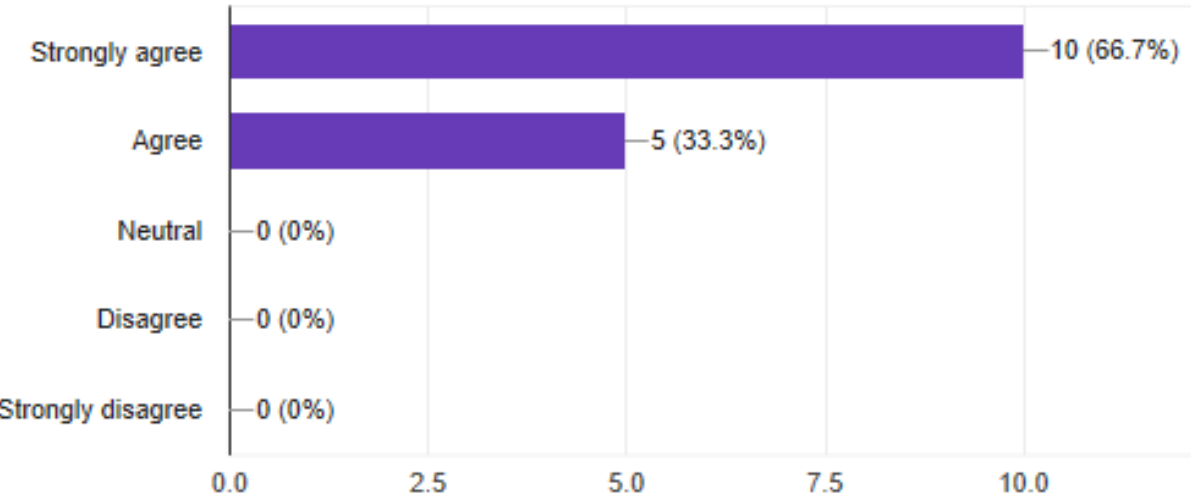

Course content: There was sufficient hands-on practical time.

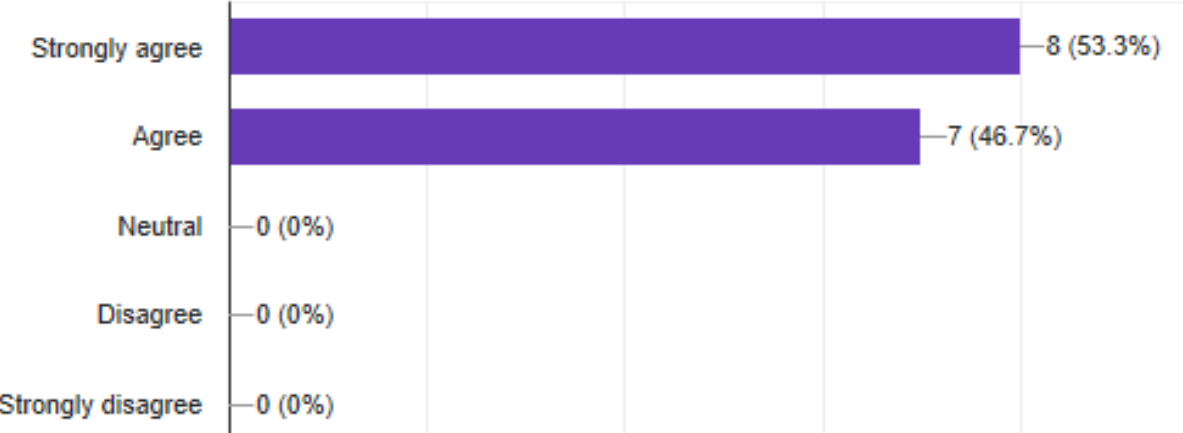

Course content: I feel more confident about performing the regional nerve blocks I learned today

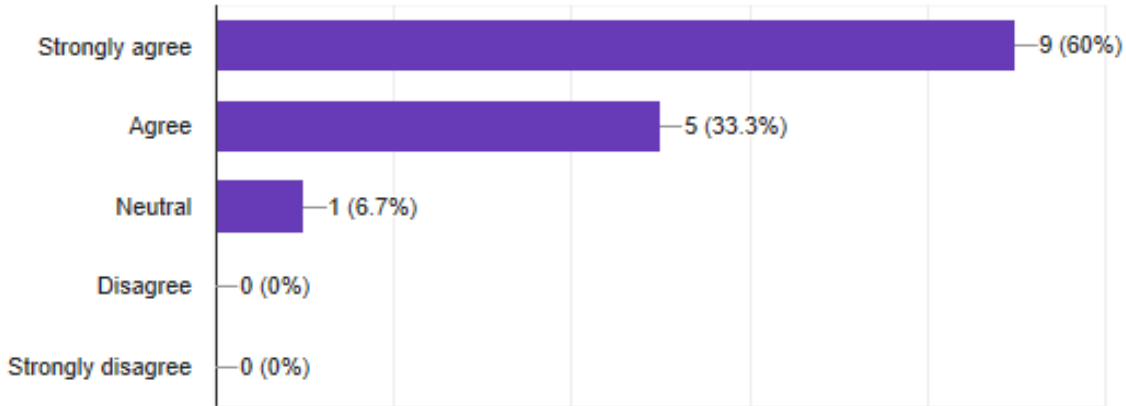

Course content: I would recommend a colleague to this course

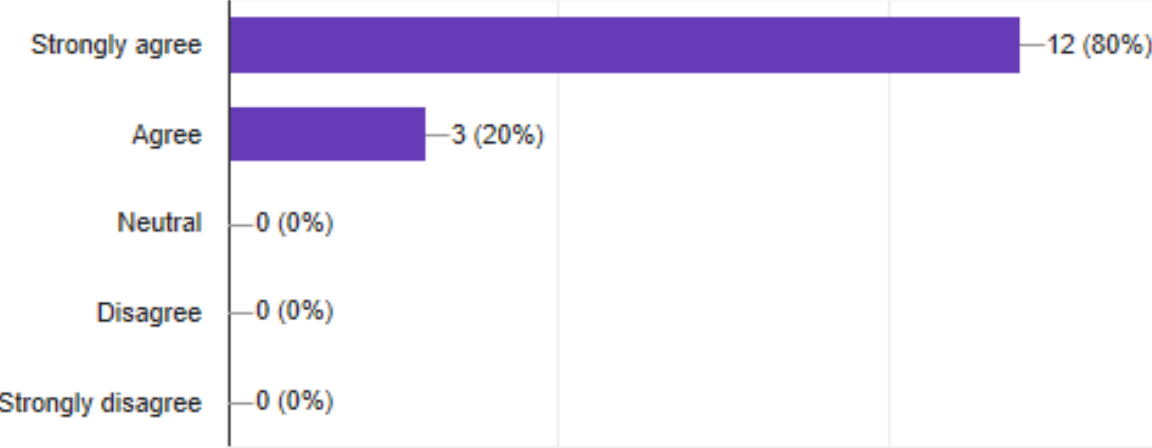

How do you think this course has changed your practice?

- Feel more confident in doing blocks • More confident with scanning • Improved knowledge of workings of USS machine - more inclined to play with settings - Much more confident with USS use and blocks • Improved confidence in a variety of blocks. - Lots of practice on other people - Given me more confidence to try blocks, and seek out lists with blocks to improve my practice - Would be more confident to practice blocks independently - Confidence - More confident of indications for peripheral blocks - Improved coordination, and ability to problem solve if images of nerves poor - Confidence in options for using different blocks $•$ More confidence with blocks $•$ Better confidence

\section{Conclusion}

Universal agreement on the most effective way to teach ultrasound guided regional anaesthesia has still not been reached. ${ }^{1}$ However, we believe that there is a need for a cost effective intermediate level workshop for anaesthetic trainees. We have demonstrated that with the right planning and dedicated experienced faculty, this can be delivered efficaciously. 